\title{
Long-term fate of terminally differentiated skeletal muscle cells following E1A-initiated cell cycle reactivation
}

\author{
L Latella $^{1,2}$, A Sacchi ${ }^{1}$ and M Crescenzi ${ }^{\star, 1,2}$ \\ ${ }^{1}$ Molecular Oncogenesis Laboratory, Regina Elena Cancer Institute, Rome, Italy \\ ${ }^{2}$ Laboratory of Comparative Toxicology and Ecotoxicology, Istituto Superiore di \\ Sanita', Rome, Italy \\ * Corresponding author: Marco Crescenzi, Istituto Superiore di Sanita', Viale \\ Regina Elena, 299, 00161 Roma, Italy. Tel: +39 0649903163; \\ Fax: +39 0649902355; E-mail: crescenz@iss.it
}

Received 29.6.99; revised 20.8.99; accepted 27.8.99

Edited by R Knight

\begin{abstract}
We have previously shown that E1A reactivates the cell cycle in 'irreversibly' growth arrested, terminally differentiated (TD) cells. The molecular events following E1A-mediated reactivation of TD skeletal muscle cells have been extensively investigated. However, the long-term fate of the reactivated cells has not been directly determined. In this paper, E1A is used to reactivate TD myotubes derived from established cell lines or primary myoblasts. We show that the reactivated muscle cells continue proliferating beyond the end of the first cell cycle and progress through at least a second one. Experiments performed with an inducible E1A/estrogen receptor chimera indicate that the reactivated cell cycle is self-sustained, since E1A is exclusively necessary to reactivate TD cells, but is dispensable for both the continuation of the first cycle and the progression into the following one. Finally, we report that E1A-mediated reactivation of muscle cells results in apoptotic cell death that can be delayed by the antiapoptotic, adenoviral E1B $55 \mathrm{kDa}$ oncogene. Cell Death and Differentiation (2000) 7, 145-154.
\end{abstract}

Keywords: terminal differentiation; mitosis; cytokinesis; time-lapse videomicroscopy

Abbreviations: TD, terminally differentiated; MSC, mouse satellite cells; Ara-C, $\beta$-D-arabinofuranoside; BrdUrd, 5-bromo-2'-deoxyuridine; $\mathrm{MHC}$, myosin heavy chain; PI, post infection; TUNEL, terminal deoxyribonucleotide transferase-mediated dUTP nick end labeling; $\mathrm{SF}$, serum free

\section{Introduction}

Terminally differentiated cells are defined by specialized properties, which are the result of tissue-specific gene expression, associated with a physiologically irreversible growth arrest. Terminal differentiation characterizes the majority of the cells in an adult mammal. The inability of TD cells to replicate poses strategic problems to the organism.
These cells must live as long as the organism itself, which requires extraordinary measures to ensure their survival. In addition, organs and systems devoid of stem cell compartments and whose parenchyma is composed exclusively of TD cells cannot renew their tissues by means of cell proliferation. This makes them especially vulnerable to irreplaceable cell loss caused by diseases or injuries. The ability to induce sustained proliferation of TD cells such as neurons, myocardiocytes, or endocrine cells would potentially have a major impact on the therapy of diseases and traumas of such organs.

One example of terminal differentiation is provided by skeletal muscle cells. ${ }^{1}$ The differentiation process of these cells can be recapitulated in vitro. Primary as well as established myoblasts can be cultured extensively. Mitogen withdrawal starts the differentiation program, which begins with irreversible exit from the cell cycle (commitment). Postmitotic myoblasts then express muscle-specific genes, turning into mononucleated myocytes (biochemical differentiation). The final stage of skeletal muscle differentiation in vitro involves fusion of myocytes into multinucleated structures called myotubes (morphological differentiation). ${ }^{1}$

It has been shown that a number of DNA tumor virus oncogenes are able to reactivate the cell cycle in TD cells. $^{2-6}$ Adenoviruses bear the E1A oncogene, which shares this ability. ${ }^{6}$ Adenoviruses are particularly well suited for cell cycle reactivation of TD cells, since they can infect almost all cell types ${ }^{7,8}$ across a wide range of species, ${ }^{6}$ irrespective of the proliferation status of the cells. In fact, we and others have shown that adenovirus infection and the consequent $\mathrm{E} 1 \mathrm{~A}$ expression can reactivate a variety of TD cells, including myotubes, adipocytes, ${ }^{6,9}$ myocardiocytes, ${ }^{10}$ and neurons (M Crescenzi, unpublished). E1A exerts such activity by activating genes normally expressed at the G1/S boundary. ${ }^{11}$ By this mechanism, E1A in effect bypasses the cell cycle block that prevents TD myotubes from proceeding beyond midG1 phase upon mitogenic stimulation. ${ }^{12}$

We have shown that E1A-reactivated myotubes undergo DNA replication, mitosis, and cytokinesis. ${ }^{6,9}$ However, the long-term fate of these cells has not been investigated in depth. Open questions include whether further cell cycles follow the first one, whether any fraction of the reactivated cells can survive indefinitely, and whether the activity of $\mathrm{E} 1 \mathrm{~A}$ is required to sustain the cell cycle or it is only needed to initiate it. All of these issues are relevant in view of potential therapeutic applications.

In this paper, we present evidence that TD skeletal muscle cells reactivated by $\mathrm{E} 1 \mathrm{~A}$ undergo more that one cell cycle. We show that the reactivated cells eventually die by apoptosis. Such outcome can be at least partially opposed by the antiapoptotic adenovirus E1B oncogene, which allows extended survival. Finally, making use of a 
conditional E1A chimera, we show that the cell cycle activated by the oncogene in myotubes is auto-maintained and, for its continuation, can dispense with the functional presence of E1A.

\section{Results}

\section{Mitotic phenomena associated with cell cycle reactivation in myotubes}

As already described, adenovirus-transduced E1A reactivates DNA synthesis in myotubes derived from established and primary myoblasts as well. While DNA synthesis occurs in E1A-reactivated myotubes irrespective of the presence of growth factors, the successive mitosis is greatly facilitated by serum. ${ }^{6}$ Multinucleated myotubes undergo mitosis in a quasi-synchronous fashion. In elongated myotubes, whose nuclei are distributed over a long distance, mitosis often appears to initiate in a single nucleus. A mitotic 'wave' then ensues, probably due to the diffusion of regulatory factors from one nucleus to the next. The result is the simultaneous presence of nuclei arranged in an orderly progression of mitotic stages (Figure 1a,b). Mitosis is accompanied by cytokinesis, which may or may not result in successful cell division. As shown in Figure 1, mitotic myotubes attempt to divide. Time-lapse videomicroscopy shows that myotubes sometimes separate into two or more fragments (Figure 2), sometimes stretch but eventually recollapse into a single structure. Most mitoses present morphological aberrations ranging from minor anomalies to gross disruption of nuclear organization. These include chromosomal displacement, incomplete chromatid disjunction, extensive micronucleation, and coalescence of multiple nuclei into a giant one (Figure 1).
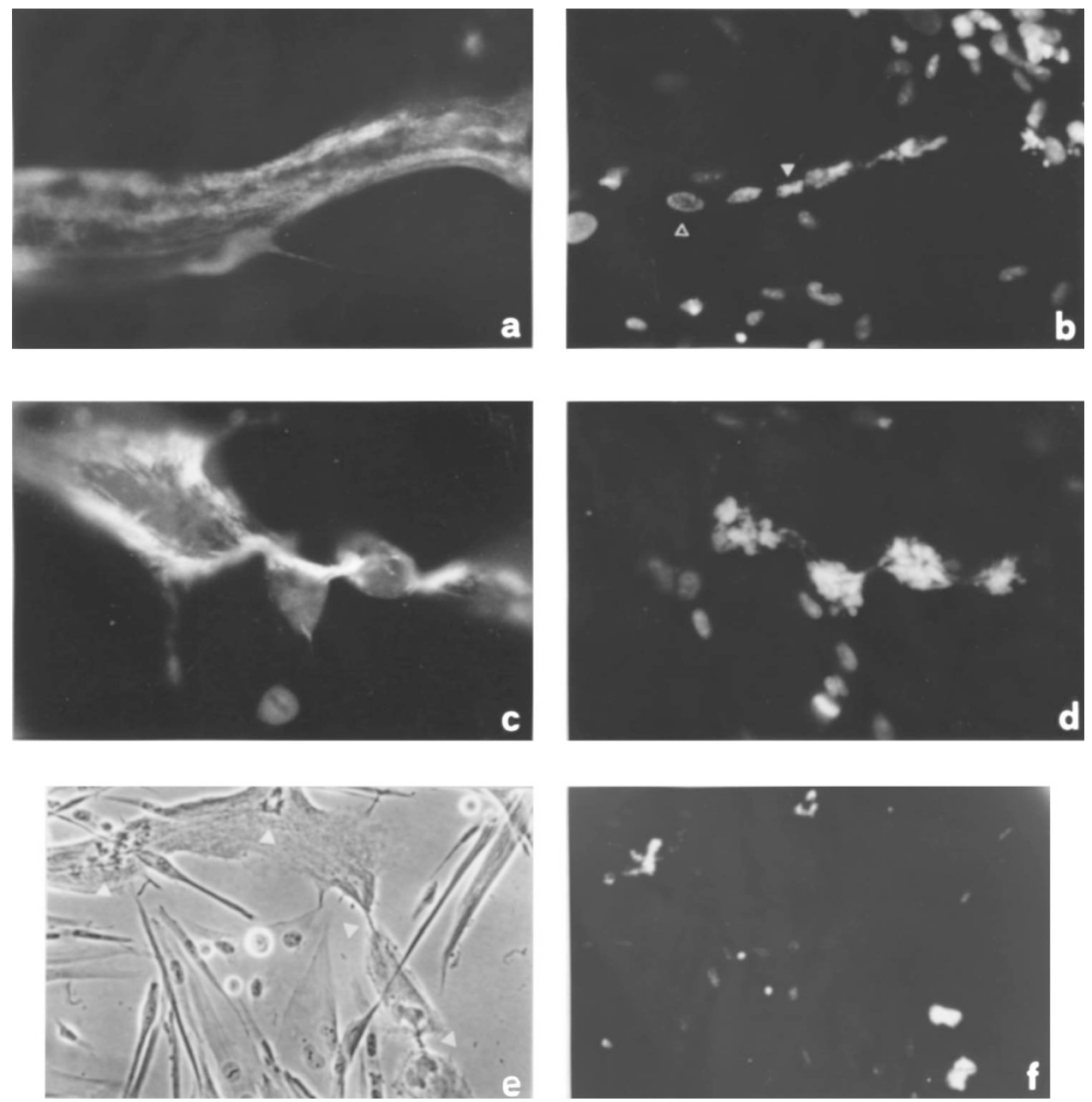

Figure 1 Mitotic events in E1A-reactivated myotubes. Myotubes derived from primary mouse satellite cells were infected with dl520 and subjected to immunofluorescence for MHC (a,c), stained with Hoechst $33258(\mathbf{b}, \mathbf{d}, \mathbf{f})$, or photographed in phase contrast (e). Top panels: a MHC-positive myotube (a) shows an orderly progression of mitotic stages. The leftmost nucleus in (b), only partially included in the picture, is in interphase; the next one, (open triangle) in prophase; the next one in prometaphase; the next one (closed triangle) in metaphase; the next two are daughter nuclei in anaphase. Notice that no cleavage furrow is visible in (a). Middle panels: a MHC-positive myotube (c) shows multiple cleavage furrows enclosing mitotic nuclei. The size of the two largest nuclear masses in (d) suggests that they result from the coalescence of multiple nuclei. Bottom panels: part of a large, mitotic myotube is shown in (e), while (f) is the corresponding Hoechst 33258 staining. The four triangles in (e) indicate, from left to right: an area with several mitotic nuclei; a tripolar anaphase; a cleavage furrow not corresponding to any mitotic nuclei; a cleavage furrow separating two nuclear masses (f) presumably derived from the fusion of multiple nuclei 


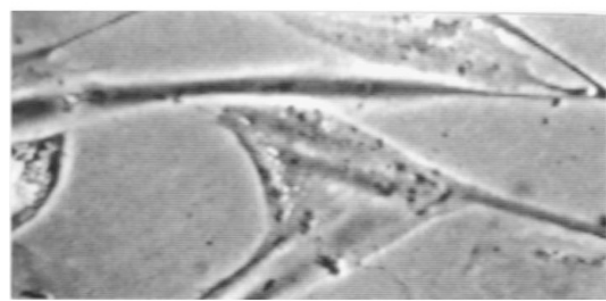

$\underline{\text { hr PI }}$

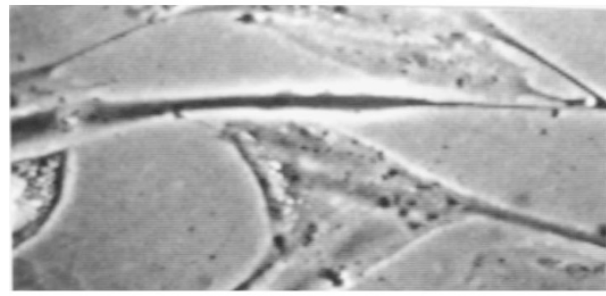

$36: 52$

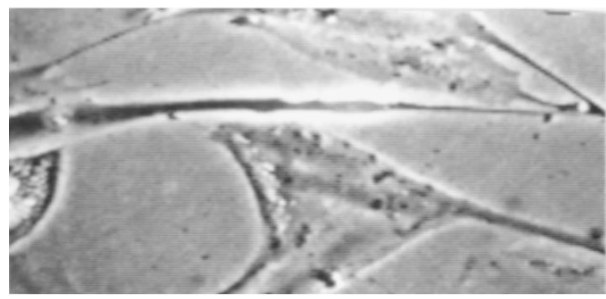

$37: 09$

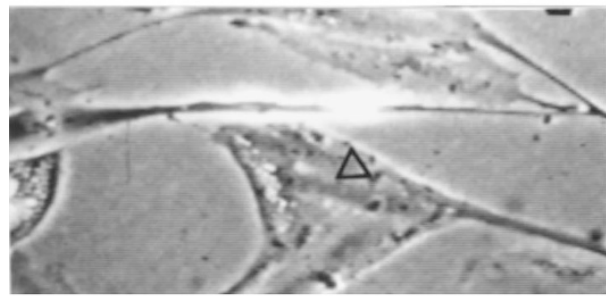

$37: 16$

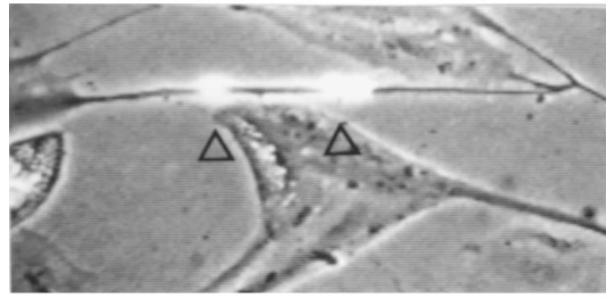

$37: 29$

$38: 38$

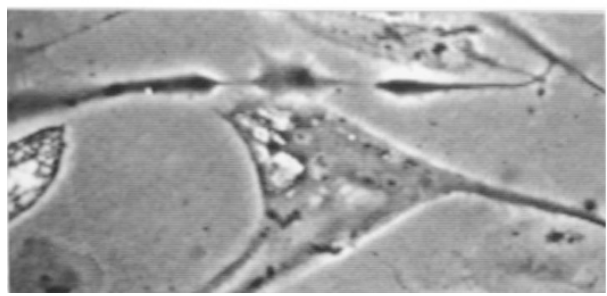

$41: 08$

Figure 2 Time-lapse videomicroscopy of a dividing myotube. Myotubes derived from quail embryo myoblasts were infected with dl520 and subjected to videomicroscopy; selected frames are shown and time elapsed from infection (hours: minutes) is indicated. A branch of a large myotube, initially relatively flat (first frame from top) begins to bulge as it enters mitosis (second and third frames). In the fourth frame, a section of the myotube including one of the nuclei rounds up and becomes highly refringent in phase contrast (indicated by a triangle). In the fifth frame, a second nucleus also rounds up,

\section{E1A-reactivated myotubes undergo multiple cell cycles}

A question not directly addressed in our previous studies is whether E1A can induce TD myotubes to go through more than one cell cycle. To answer this question, we performed cell cycle analyses of TD muscle cells at different times after infection with the mutant dl520 adenovirus. Since myotubes, which possess variable numbers of nuclei, cannot be analyzed by cytofluorimetry, for these studies we used primary myocytes derived from murine satellite cells (MSC). TD, mononucleated myocytes were obtained by sparsely seeding MSC before induction of differentiation, so as to prevent fusion of the majority of them. Undifferentiated myoblasts that might have altered the results of the analyses were eliminated by $\beta$-D-arabinofuranoside (Ara-C) treatment. Figure 3 shows a time-course analysis of an experiment in which $68 \%$ of the myocytes were reactivated by dl520 infection, as determined by 5-bromo-2'-deoxyuridine (BrdUrd) staining. DNA synthesis began at approximately $36 \mathrm{~h}$ post infection $(\mathrm{PI})$ and was fully under way at $42 \mathrm{~h}$. The first cell cycle was essentially complete at $48 \mathrm{~h} \mathrm{PI}$, when most of the reactivated cells were in G2/M phase or had already entered the G1 phase of the second cycle. By $54 \mathrm{~h} \mathrm{PI}$, a second S-phase was evidently taking place, with a relevant fraction of the cells reaching G2/M phase for the second time by $72 \mathrm{~h}$. Indeed, at this time a significant number of octaploid cells appeared, presumably being the result of DNA replication followed by ineffective cell division. Between 72 and $86 \mathrm{~h} \mathrm{PI}$, the reactivated cells began to die, giving raise to a hypodiploid peak, reminiscent of apoptosis. At $130 \mathrm{~h} \mathrm{PI}$, the peaks of DNA content unambiguously corresponding to reactivated cells (the tetra- and octaploid peaks) contained fewer cells than at earlier times, suggesting that most reactivated cells had died. As expected, the cell cycle profiles of myotubes infected with the control dl312 virus or mockinfected did not change throughout the experiment.

To confirm that E1A-reactivated muscle cells can go through more than one cell cycle, we used the previously described C2(E1A-ER) cells. ${ }^{12}$ These cells are a clone of the $\mathrm{C} 2 \mathrm{C} 12$ myoblast cell line stably expressing an estrogen-inducible E1A/estrogen-receptor chimera. C2(E1A-ER) myoblasts were induced to differentiate in the absence of estrogen and in the presence of Ara-C. When the derived myotubes were exposed to estrogen, about $80 \%$ of them incorporated BrdUrd as assessed by immunofluorescence staining (see Materials and Methods). Time-course analysis of BrdUrd incorporation showed that the first DNA synthesis took place entirely between 10 and $36 \mathrm{~h}$ after estrogen addition ( ${ }^{12}$ and data not shown). Thus, to demonstrate that the $\mathrm{C} 2(\mathrm{E} 1 \mathrm{~A}-\mathrm{ER})$ cells could undergo a second cell cycle, we incubated the estrogen-reactivated

about $70 \mathrm{~s}$ after the first one. The sixth frame shows that, upon exit from mitosis, the resulting myotube fragments spread again onto the substrate. The original myotube branch is now divided into three segments. Presumably, the middle segment contains one of the daughter nuclei derived from the division of each of the two parental nuclei 
myotubes with BrdUrd between 6 and $40 \mathrm{~h}$ or 40 and $53 \mathrm{~h}$ from estrogen induction. During the first $40 \mathrm{~h}, 82 \%$ of the myotubes incorporated BrdUrd, while $64 \%$ of them synthesized DNA during the second, shorter interval. These results indicated that at least $3 / 4$ of the myotubes that had been initially reactivated went through a second $S$ phase. To show that BrdUrd incorporation during the second interval was not ascribable to an indefinitely protracted S-phase, identical dishes of C2(E1A-ER) myotubes were induced with estrogen and subjected to serial, 6-h pulses of BrdUrd. Figure 4 shows that BrdUrd incorporation occurred in two distinct phases, with peaks at 30 and $48 \mathrm{~h}$ from the beginning of estradiol induction, separated by a distinct 'minimum' at $42 \mathrm{~h}$. We conclude
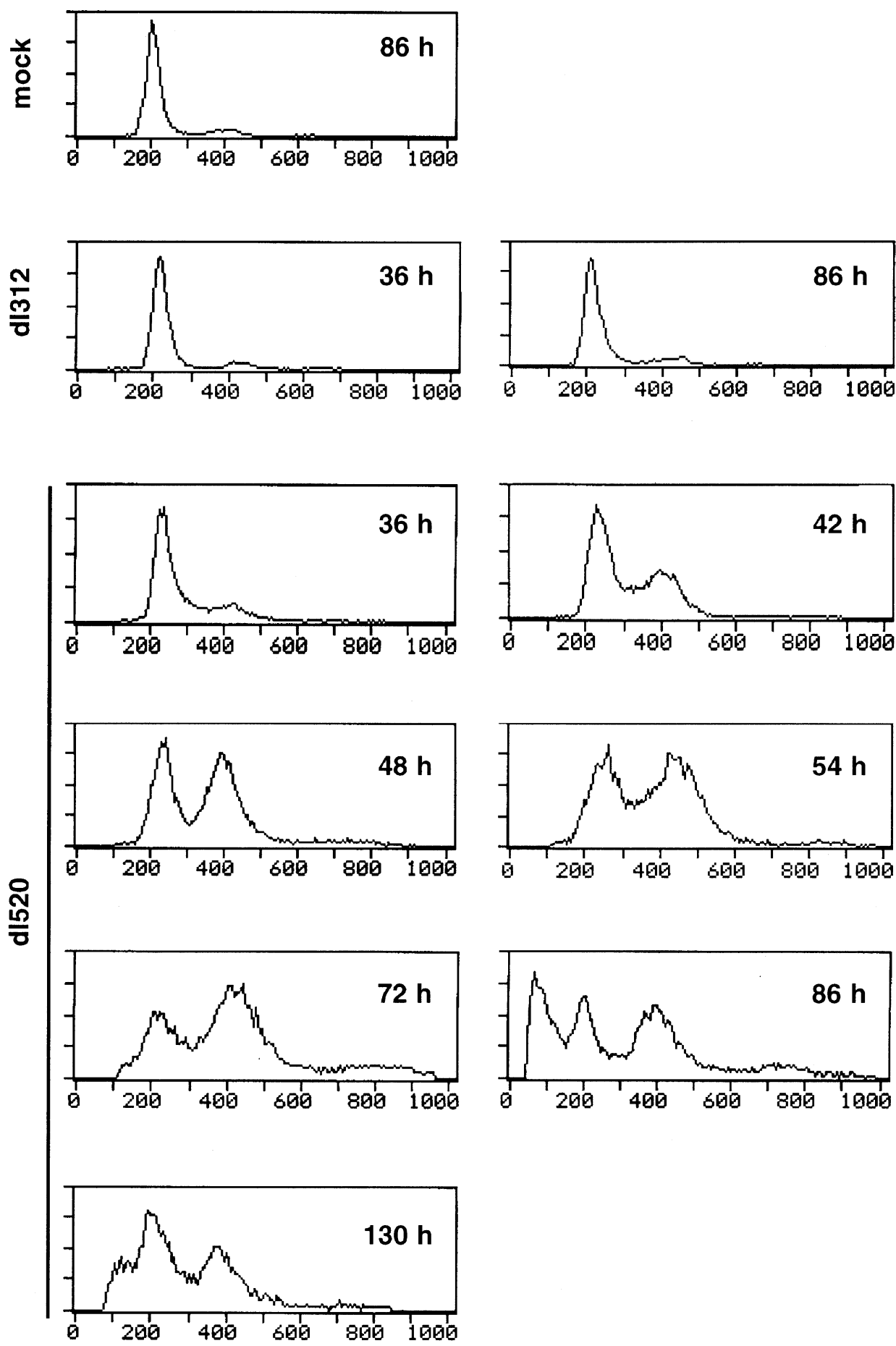

Figure 3 Cytofluorimetric cell cycle analysis of TD myocytes. Cultures of mononucleated myocytes derived from primary MSC were infected with the E1Aexpressing dl520 virus, the control dl312 mutant, or mock-infected and analyzed at the indicated times after infection 


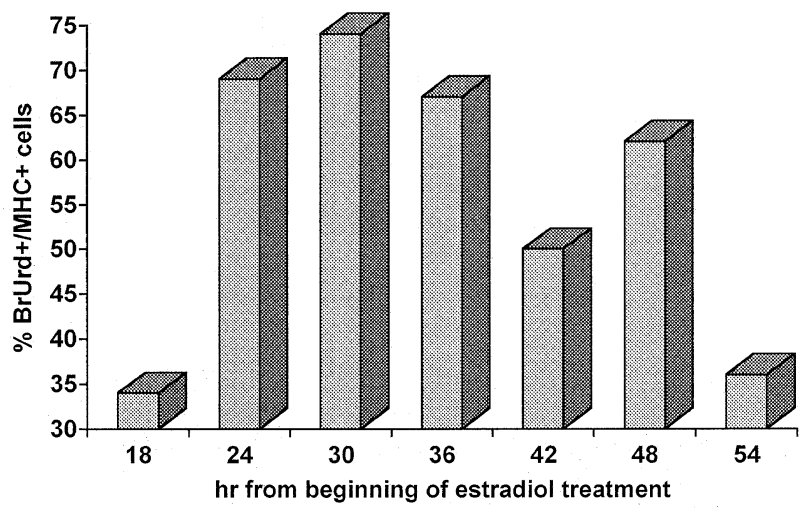

Figure 4 Time-course analysis of BrdU incorporation in C2(E1A-ER) myotubes. Parallel dishes of $\mathrm{C} 2(\mathrm{E} 1 \mathrm{~A}-\mathrm{ER})$ myotubes were stimulated with $1 \mu \mathrm{M} \beta$-estradiol, pulsed with BrdUrd at $6 \mathrm{~h}$ intervals, and double-stained for $\mathrm{MHC}$ and BrdUrd (see Materials and Methods). Labels on the abscissa refer to the end of each consecutive 6-h interval (e.g., 18 indicates the $12-18 \mathrm{~h}$ interval)

that the two peaks of BrdUrd incorporation represent two independent S-phases, separated by approximately $18 \mathrm{~h}$.

Altogether, these results show that TD muscle cells, once reactivated by $\mathrm{E} 1 \mathrm{~A}$, do not meet further blocks in their proliferation.

\section{E1A-mediated reactivation of TD muscle cells leads to eventual cell death}

In myotube cultures reactivated by $E 1 A$, cell death follows reactivation about 4 days after dl520 infection. To investigate the nature of such death, we analyzed reactivated myocytes by cytofluorimetry. A logarithmic scale for DNA content was chosen, which emphasizes the presence of a hypodiploid DNA peak, typical of apoptotic cell death (Figure 5). At $96 \mathrm{~h}$ PI, control dl312-infected myocytes showed a relatively modest hypodiploid peak. In contrast, such peak represented more than two thirds of the dl520-reactivated myocytes.

To confirm that the reactivated muscle cells undergo apoptosis, dl520- and control-infected myotubes were subjected to TUNEL analysis. Figure 6 shows cytocentrifuge preparations of infected myotubes stained with the TUNEL method. Myotubes infected with the dl520 virus became weakly positive at $48 \mathrm{~h}$ PI. Massive, TUNELpositive cell death took place by $96 \mathrm{~h} \mathrm{PI}$, consistent with the cytofluorimetric results presented in Figure 3 . Thus, we conclude that E1A-mediated cell cycle reactivation of TD muscle cells leads to apoptotic cell death within 4-5 days PI.

In an initial attempt at preventing apoptosis, we introduced into the $\mathrm{C} 2 \mathrm{Q} 7.2$ subclone of $\mathrm{C} 2 \mathrm{C} 12$ cells the $55 \mathrm{kDa}$ product of the antiapoptotic adenovirus oncogene $\mathrm{E} 1 \mathrm{~B}$, which binds and inactivates the cellular factor p53. One clone, C2Q7.2(CMVE1B55), which constitutively expresses E1B $55 \mathrm{kDa}$, was isolated (see Materials and Methods for details). Myotubes derived from this clone or the parental cells were infected with dl520 at an $\mathrm{MOI}$ of 60 , which reactivated about $50 \%$ of the differentiated cells. The
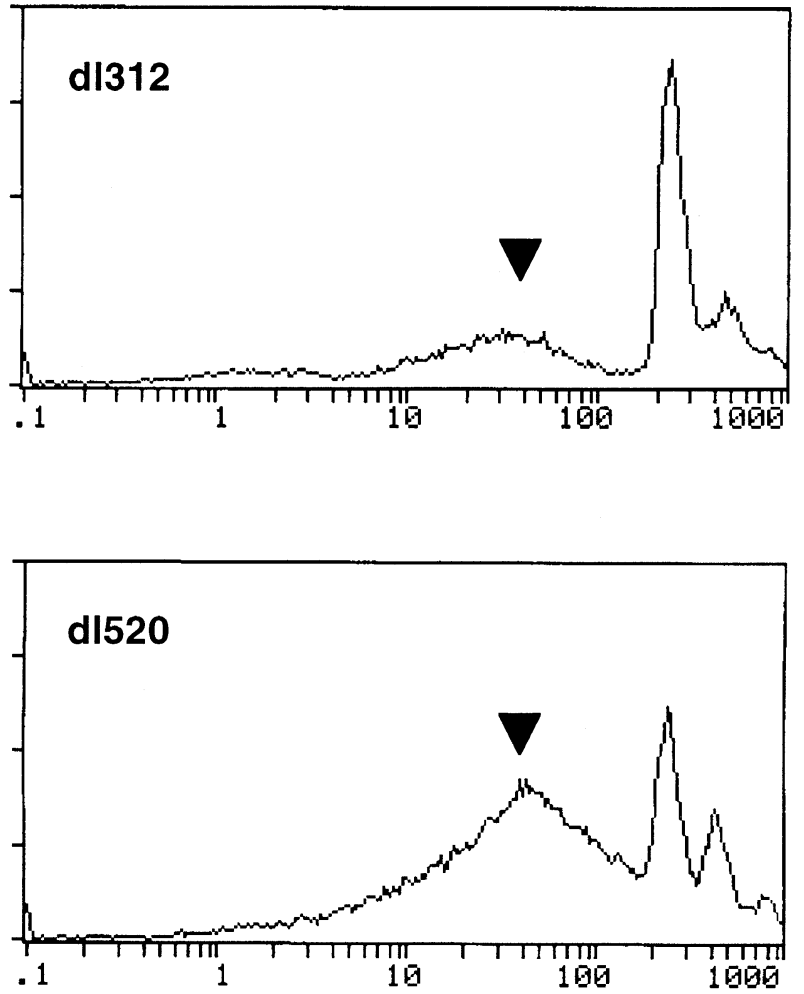

Figure 5 Hypodiploid peaks of DNA content in adenovirus-infected myocytes. MSC-derived myocytes were infected with the dl312 or dl520 virus and analyzed by flow cytometry at $96 \mathrm{~h} \mathrm{PI}$. A relatively small hypodiploid peak, marked by a closed triangle, is visible in dl312-infected myocytes, and it is greatly enhanced in the dl520 cells

infected myotubes were TUNEL stained at $120 \mathrm{~h} \mathrm{PI}$. At this time, most parental cells were TUNEL-positive, while the E1B-expressing cells were almost entirely negative (Figure 7).

To determine whether in these cells resistance to apoptosis is associated with prolonged survival, dl520infected C2Q7.2(CMVE1B55K) myocytes were followed by time-lapse videomicroscopy. Remarkably, the cells survived up to 7 days after infection and underwent up to three mitoses (data not shown). However, even these partially resistant cells eventually died by apoptosis, as indicated by TUNEL staining at 7 days PI (data not shown).

\section{E1A is not required to sustain the reactivated cell cycle in myotubes}

Since the cell cycle is believed to be directed by sequential events that regulate one another, we conjectured that $\mathrm{E} 1 \mathrm{~A}$ might be needed in TD cells only for cell cycle initiation, but not for its maintenance. To test this hypothesis, we exploited again the $C 2(E 1 A-E R)$ myoblasts. About $80 \%$ of the myotubes derived from these cells can be reactivated by the continuous presence of $1 \mu \mathrm{M} \beta$-estradiol. We determined the shortest pulse of $\beta$-estradiol still sufficient to reactivate a significant fraction of the cells. It was found that a pulse as short as $6 \mathrm{~h}$, followed by extensive washes, could induce DNA synthesis in about $30 \%$ of the myotubes. In this setting, 

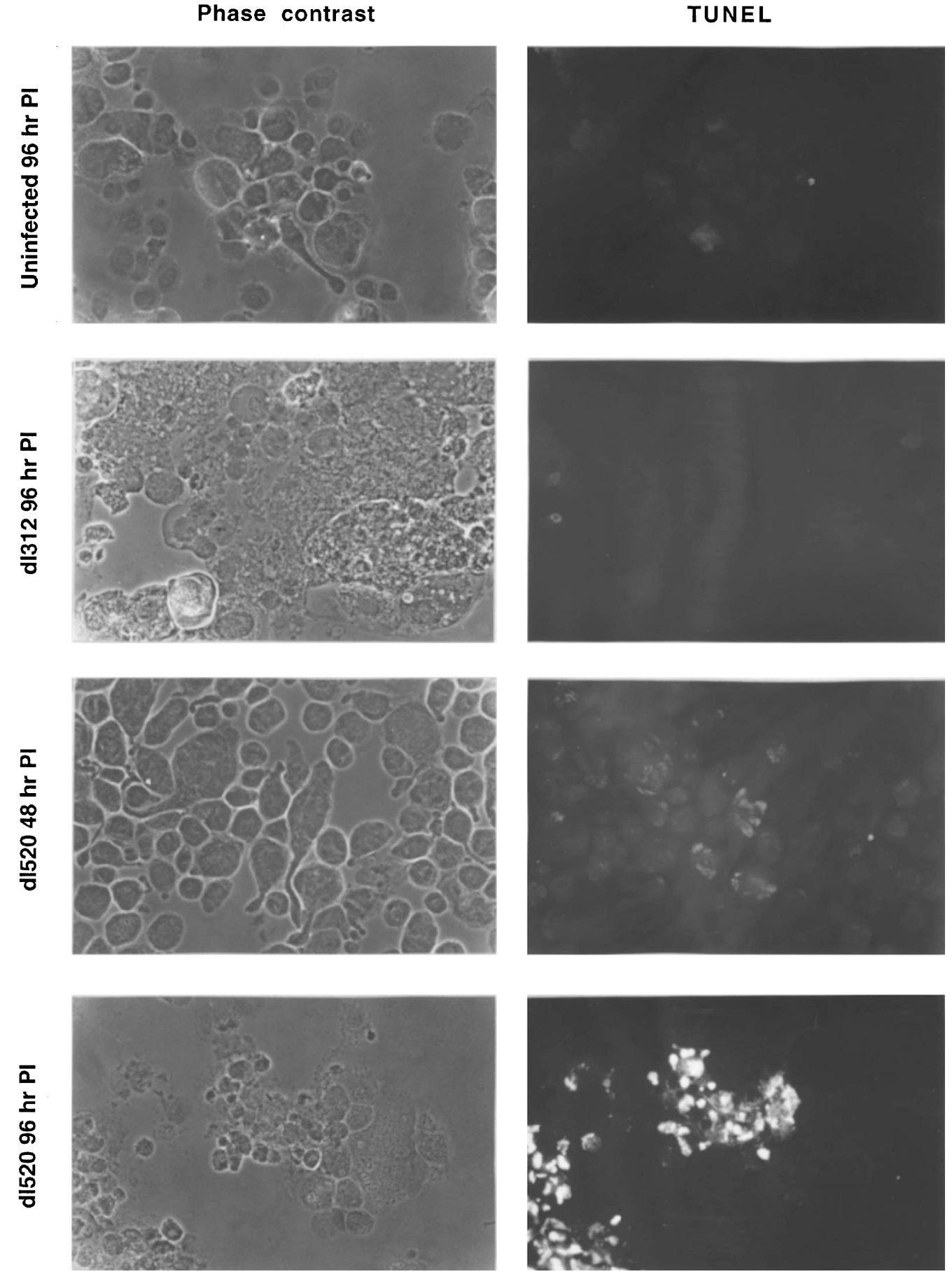

Figure 6 TUNEL staining of infected myocytes. MSC-derived myotubes were infected with the indicated viruses and cytocentrifuge slides, inclusive of any detached cells, were prepared at the indicated times. dl520-infected myocytes were slightly TUNEL-positive at $48 \mathrm{~h} \mathrm{PI}$ and mostly positive at $96 \mathrm{~h}$. Uninfected and dl312-infected myocytes remained TUNEL-negative even at $96 \mathrm{~h} \mathrm{PI}$

S-phase starts no earlier than $10 \mathrm{~h}$ after the beginning of the estrogen pulse and at least $4 \mathrm{~h}$ after its end. ${ }^{12}$ After $24 \mathrm{~h}$ from the beginning of stimulation, mitotic figures began to appear, demonstrating continuation of the cell cycle in the absence of $\beta$-estradiol. To determine whether the pulse-reactivated myotubes proceeded into a second cell cycle, we subjected them to BrdUrd pulse labeling. Replica dishes of C2(E1A-ER) myotubes stimulated from 0 to $6 \mathrm{~h}$ with $\beta$-estradiol were incubated with BrdUrd from 6 to $40 \mathrm{~h}$ or from 40 to $53 \mathrm{~h}$. The intervals were chosen considering that there is no increase in the percentage of BrdUrd-positive cells after $36 \mathrm{~h}$ from the beginning of estrogen stimulation (data not shown). This indicates that the first S-phase is completed by $36 \mathrm{~h}$ and that BrdUrd incorporation occurring after that time is the result of 


\section{Phase contrast}
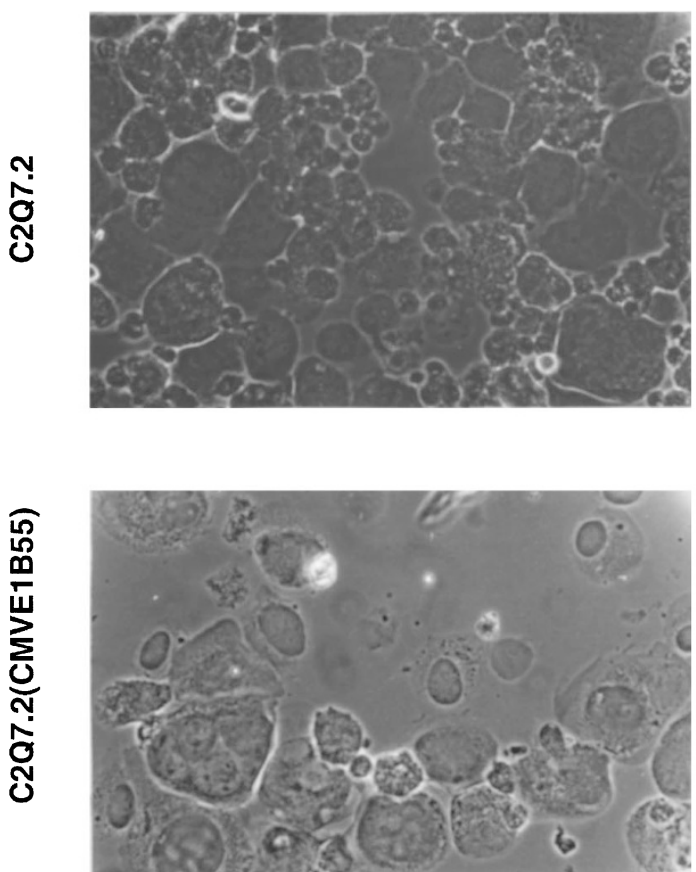

TUNEL
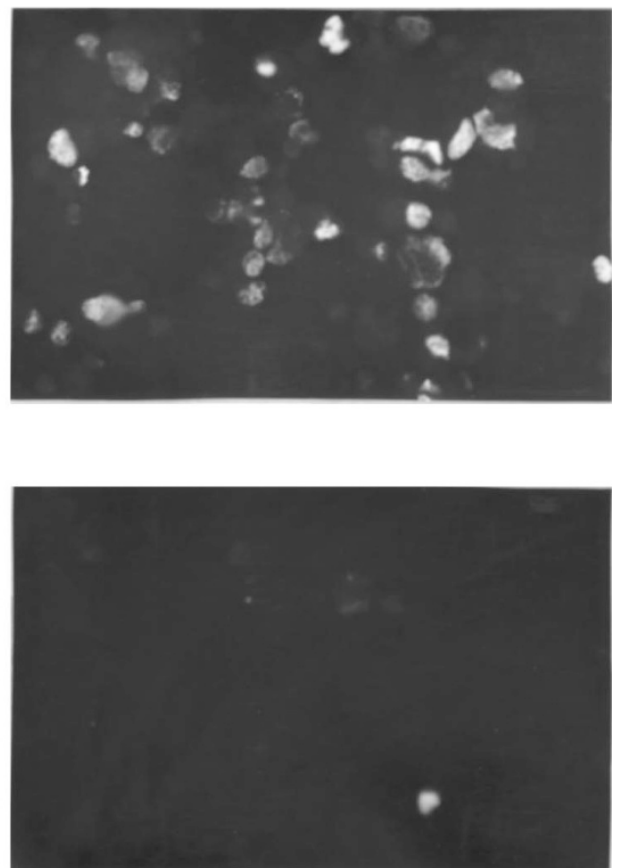

Figure 7 Expression of E1B $55 \mathrm{kDa}$ affords partial protection from dl520-induced apoptosis. Myotubes derived from the C2Q7.2(CMVE1B55) clone and the parental C2Q7.2 cells were infected with dl520. Cytospin preparations were TUNEL stained at $120 \mathrm{~h} \mathrm{PI}$. At this time, the parental cells were mostly TUNEL-positive, while the E1B-expressing cells remained essentially negative

new DNA synthesis in cells that have already gone through Sphase at least once. Table 1 compares C2(E1A-ER) myotubes continuously stimulated with $\beta$-estradiol with those pulsed for only $6 \mathrm{~h}$. In both instances, the majority of the cells labeled in the first interval underwent a second round of DNA replication between 40 and $53 \mathrm{~h}$. These data show that continuous estradiol stimulation, presumptively corresponding to $E 1 A$ activation, is not necessary for cell cycle progression. In an experiment similar to that shown in Figure 4, BrdUrd incorporation was assessed at 6-h intervals in C2(E1A-ER) myotubes stimulated with estradiol for $6 \mathrm{~h}$. The myotubes incorporated BrdUrd in two phases that peaked at 30 and $\geqslant 54 \mathrm{~h}$ from the beginning of estradiol induction (Figure 8a). The two phases were separated by a gap at $42 \mathrm{~h}$. The first peak of BrdU incorporation and the gap occurred at time points closely matching those of continuously stimulated myotubes, while the second incorporation phase was somewhat more extended in time. Again, we conclude that the two incorporation peaks represent two, separate S-phases belonging to successive cell cycles.

To verify that estrogen withdrawal indeed causes complete E1A inactivation, we exploited differentiation as an indicator of E1A activity. Skeletal muscle differentiation is a cellular competence exquisitely sensitive to the activity of virtually all defined E1A functional domains. ${ }^{11,13-16} \mathrm{We}$ reasoned that if any residual E1A activity were left after estradiol removal, it would significantly impair differentiation of myoblasts transiently stimulated with estrogen. Three identical cultures of undifferentiated C2(E1A-ER) myoblasts were induced to differentiate after diverse treatments. One
Table 1 Reactivation of C2(E1A-ER) myotubes

\begin{tabular}{lcc}
\hline & \multicolumn{2}{c}{$\%$ BrdUrd+ myotubes } \\
& Continuous estradiol & $\mathbf{6}$ h estradiol pulse \\
\hline $6-40 \mathrm{~h}$ & 82 & 30 \\
$40-53 \mathrm{~h}$ & 64 & 18 \\
\hline
\end{tabular}

culture was continuously treated with $\beta$-estradiol for $11 \mathrm{~h}$ in growth medium; differentiation was induced thereafter with serum-free medium containing $\beta$-estradiol. A second culture was stimulated with estradiol for $6 \mathrm{~h}$ and then washed free of estrogen for $5 \mathrm{~h}$ before inducing differentiation in the absence of estrogen. Finally, a third cell population was not stimulated with $\beta$-estradiol before or after inducing differentiation. The cultures were evaluated 4 days after induction of differentiation. As expected, cells constitutively treated with $\beta$-estradiol did not survive serum-free induction, presumably dying by E1A-triggered apoptosis. The extent of differentiation in the two surviving cell populations was then compared. The number of myosin heavy chain (MHC)-expressing (differentiated) cells was similar: by immunofluorescence, the pulse-treated and the untreated cultures showed 2.06 and 2.75 MHC-positive cells per highpower microscopic field, respectively. To eliminate the possible influence of cell death in the assessment of differentiation, we determined by Western blotting the total amount of $\mathrm{MHC}$ expressed by the two cultures, including the floating, dead cells. If residual E1A activity caused cell death or otherwise prevented differentiation of transiently- 
a

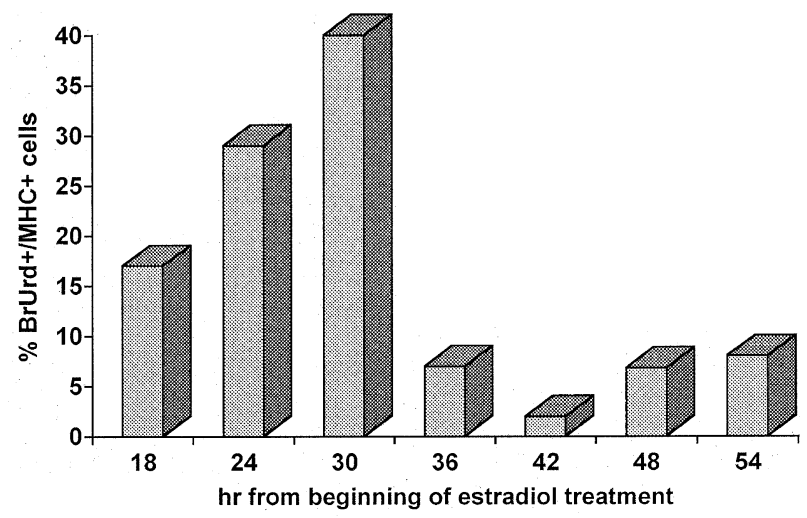

b

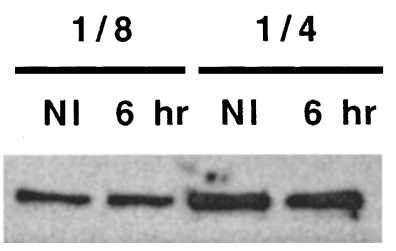

c

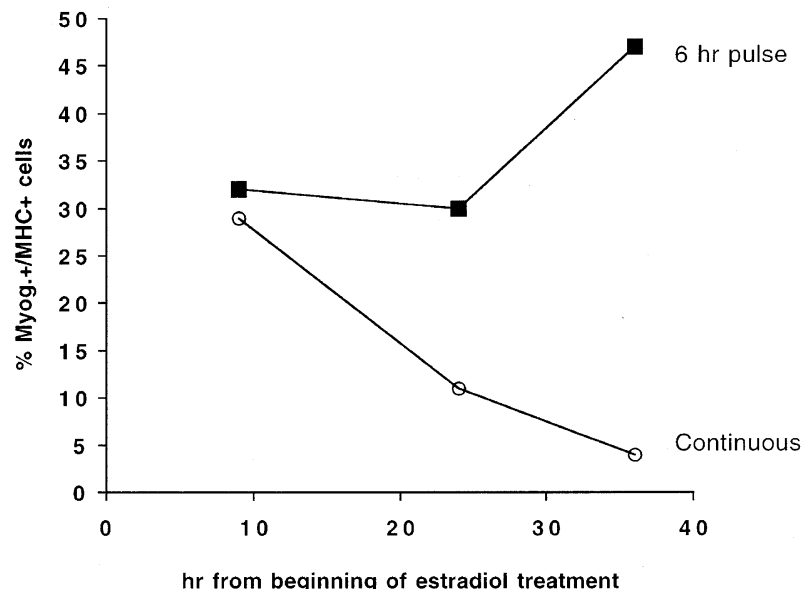

Figure 8 Six-h activation of C2(E1A-ER) cells. (a) C2(E1A-ER) myotubes were treated for $6 \mathrm{~h}$ with $1 \mu \mathrm{M} \beta$-estradiol, then washed and cultured in the absence of estrogen; time-course analysis of BrdU incorporation as in Legend to Figure 4. (b) Western blot analysis of MHC expression in $\mathrm{C} 2(\mathrm{E} 1 \mathrm{~A}-\mathrm{ER})$ myotubes. C2(E1A-ER) myoblasts were either not induced or induced with $1 \mu \mathrm{M} \beta$-estradiol for $6 \mathrm{~h}$ in growth medium, washed, and cultured for further $5 \mathrm{~h}$ in growth medium in the absence of estradiol. At the end of the latter period, both uninduced and pulse-induced cultures were switched to serum-free medium to trigger differentiation. After 4 days, protein extracts were prepared from the entire cultures, including the detached cells. Equal fractions $\left(\frac{1}{4}\right.$ or $\left.\frac{1}{8}\right)$ of the protein extracts were analyzed by Western blotting with an anti$\mathrm{MHC}$ antibody. NI: not induced; $6 \mathrm{~h}$ : pulsed for $6 \mathrm{~h}$ with $\beta$-estradiol. (c) Parallel sets of Ara-C-purified cultures of $\mathrm{C} 2(\mathrm{E} 1 \mathrm{~A}-\mathrm{ER})$ myotubes were treated for $6 \mathrm{~h}$ with $1 \mu \mathrm{M} \beta$-estradiol, then washed and cultured in the absence of estrogen ( $6 \mathrm{~h}$ pulse) or cultured in the continuous presence of $\beta$-estradiol (Continuous). At the indicated time points, cultures were double-stained for myogenin and MHC (see Materials and Methods for details). Untreated myotubes at time 0 were $88 \%$ myogenin-positive. The percentages of myogenin-positive myotubes at each time point are indicated. stimulated myoblasts, this should result in a reduction in the total MHC expressed by the entire cell population. On the contrary, the transiently stimulated and the untreated cell cultures expressed identical amounts of MHC (Figure 8b), suggesting that E1A is functionally silent already $5 \mathrm{~h}$ after $\beta$-estradiol withdrawal.

To test the reversibility of E1A-ER activation in myotubes, rather than myoblasts, we verified the effects of estrogen stimulation and withdrawal on the expression of an early differentiation marker, myogenin, by C2(E1AER) myotubes. Identical dishes of Ara-C-purified C2(E1AER) myotubes were either continuously stimulated or pulsed for $6 \mathrm{~h}$ with estrogen, washed, and maintained in estrogen-free medium. Dishes from both series were stained for myogenin and $\mathrm{MHC}$ by double, indirect immunofluorescence at 9,24 and $36 \mathrm{~h}$ from the beginning of estradiol treatment. At time $0,88 \%$ of the unstimulated myotubes were myogenin-positive. As shown in Figure 8c, myogenin-positive myotubes dropped to about $30 \%$ in both cultures at $9 \mathrm{~h}$. However, while the percentage of myogenin-positive myotubes continued to decline with time in the chronically stimulated cultures, the fall halted and then reverted in pulse-treated myotubes. Thus, the effects of E1A activation can be reversed in myotubes, at least as judged by the influence of the oncogene on myogenin expression.

Taken together, the above experiments lead to the conclusion that, in all likelihood, the functional presence of $\mathrm{E} 1 \mathrm{~A}$ is only required to initiate proliferation in TD myotubes and is no longer necessary to complete the first cell cycle or to initiate a second one.

\section{Discussion}

We have previously shown that adenovirus $\mathrm{E} 1 \mathrm{~A}$ can reactivate the cell cycle in TD myotubes and adipocytes ${ }^{6}$ and have investigated in depth the mode of action of the oncogene. ${ }^{11}$ Our preferred experimental system, the skeletal muscle, has thus shown to be well suited for basic investigations of cell cycle-related events. However, in order to use muscle cells to explore the possibility of applying cell cycle reactivation to therapy, it is fundamental to assess the long-term fate of the reactivated cells. In this paper we describe and characterize the outcome of mitotic cycle reactivation in TD myotubes.

We show here that mitoses occur in E1A-transduced myotubes, following at least the first S-phase. However, in most polynucleated myotubes, mitoses are grossly aberrant. Since many nuclei share the same cytoplasm, mitotic spindles often connect chromosomes belonging to more than one nucleus resulting, after caryokinesis, in newly formed nuclei of extremely variable DNA and chromosome content (Figure 1 and $M$ Crescenzi, unpublished observations). In addition, cleavage furrows are often misplaced in the myotubes, falling between two independently occurring nuclear divisions, rather than two daughter nuclei (Figure $1 e, f)$, as already found in other polynucleated cells. ${ }^{17}$ Nevertheless, small, polynucleated myotubes occasionally do divide successfully ${ }^{9}$ as ordinarily do mononucleated myocytes. ${ }^{6}$ Anyhow, problems deriving from the presence 
of multiple nuclei are irrelevant for most cell types other than muscle.

We report here that apoptotic cell death virtually invariably follows E1A-mediated reactivation of myotubes, as recently described for SV40 Large T-antigen. ${ }^{18}$ In principle, there might be two alternative explanations for such outcome. On one hand $E 1 A$, a known apoptosis inducer, might extrinsically trigger cellular suicide mechanisms while reactivating the cell cycle. Alternatively, apoptosis might be the intrinsic result of damage occurring as a consequence of inappropriate cell cycle reentry by a TD cell. In the first case, cell death should be avoidable by interfering with the cellular apoptotic cascade. In the second scenario, cell death would be the insuppressible effect of physical cellular damage. At this time, the first hypothesis appears more plausible, since E1B $55 \mathrm{kDa}$ is able to delay apoptosis significantly and allow more cell cycles to occur. The eventual occurrence of apoptosis even in $\mathrm{E} 1 \mathrm{~B} 55 \mathrm{kDa}$-expressing cells is not surprising in light of the fact that this protein is believed to prevent only p53dependent programmed cell death. ${ }^{19}$ Further studies are clearly necessary to determine the causes of apoptosis in reactivated TD cells and to find practical means to suppress this outcome.

The question of whether the reactivated muscle cells can undergo more than one cell cycle has been addressed in two, complementary ways. Cytofluorimetric evaluation of dl520-reactivated myocytes and analysis of C2(E1A-ER) myotubes concordantly lead to the conclusion that E1Areactivated muscle cells go through at least two cell cycles, a demonstration that, to our knowledge, has not been provided in any other TD system. ${ }^{18}$

If cell cycle reactivation of TD cells is to be exploited for therapeutic purposes it would be desirable to eventually rid the reactivated cells of the exogenous, cell cyclestimulatory gene. Using adenoviruses as transducing agents makes this purge automatic, since these viruses do not integrate their DNA into that of their hosts. Thus, in dividing cells, replication-defective adenoviral genomes are progressively diluted and eventually lost. E1A-ER pulseactivation experiments indicate that $E 1 A$ is only necessary to commence the cell cycle. Once initiated, proliferation then continues as a self-maintained cellular function, dependent only on the presence of serum factors. ${ }^{6}$ This conclusion requires the demonstration that full inactivation of E1A-ER can be achieved. We attempted to address this point in two ways. We showed that transient activation of E1A-ER in myoblasts, followed by a 5 -h inactivation in the absence of estrogen, does not detectably affect the ability of myoblasts to differentiate immediately thereafter. Since this experiment involved E1A inactivation in myoblasts, its conclusions apply to the E1A-ER chimera itself, but not necessarily to the myotube intracellular environment. To verify that the chimeric molecule can be inactivated in the relevant cellular context, $\mathrm{C} 2(\mathrm{E} 1 \mathrm{~A}-\mathrm{ER})$ myotubes were transiently treated with $\beta$-estradiol. Myogenin protein expression, initially swiftly suppressed, recovered significantly upon estrogen withdrawal. It should be stressed that both myoblast differentiation ${ }^{11,13-16}$ and myogenin expression ${ }^{11}$ have been shown to be strongly suppressed by essentially all identified E1A functional domains. In consequence, these experiments suggest that all $E 1 A$ functions are reversible in $\mathrm{C} 2(\mathrm{E} 1 \mathrm{~A}-\mathrm{ER})$ myotubes. It is nonetheless conceivable that the cell cycle is sustained, in pulse-activated myotubes, by some persistent E1A activity. Any such residual activity would exert little or no influence on myoblast differentiation and myogenin expression. It would also be unable, per se, to start the cell cycle, since the percentage of reactivated myotubes is much smaller in pulse-activated than in chronically stimulated cultures (Table 1). Although we cannot formally rule out this possibility, we regard it as unlikely.

\section{Materials and Methods}

\section{Cells and viruses}

The murine $\mathrm{C} 2 \mathrm{C} 12$ myoblast line $\mathrm{e}^{20,21}$ was cultured as previously described. ${ }^{11}$ Differentiation was induced by starving the cells in serum-free (SF) medium. Where indicated, $50 \mu \mathrm{M}$ Ara-C was added to SF medium to eliminate undifferentiated cells. Within $72 \mathrm{~h}$, Ara-Cpurified myotubes contained more than $90 \%$ of the nuclei in the culture. The $\mathrm{C} 2 \mathrm{Q} 7.2$ clone of $\mathrm{C} 2 \mathrm{C} 12$ cells was chosen among a number of randomly isolated ones for its better differentiation efficiency in comparison with $\mathrm{C} 2 \mathrm{C} 12$ parental cells. C2Q7.2 cells were transfected with the pCMV-55K plasmid (kind gift of A Zantema). Stable transfectants were selected with $\mathrm{G} 418$ and screened by Western blotting; one clone expressing high levels of $55 \mathrm{kDa}$ E1B was selected for further studies. Primary MSC and quail embryo myoblasts were isolated and cultured as described. ${ }^{12,22}$ Unless otherwise stated, all experiments involving expression of $\mathrm{E} 1 \mathrm{~A}$ in differentiated muscle cells were performed in medium containing $5-7 \%$ fetal bovine serum to promote mitosis and prevent early death. ${ }^{6}$

The dl520 mutant adenovirus expresses the 12S, but not the $13 \mathrm{~S}$ E1A mRNA. The dl312 mutant does not express E1A. These mutants and the amplification and titration protocols have been described. ${ }^{11}$

\section{Antibodies and immunofluorescence}

Antibodies used: monoclonal antibody to BrdUrd (DAKO); rabbit antiserum (G Salvatori and G Cossu) or monoclonal antibody MF2023 to MHC; monoclonal antibody F5D to myogenin ${ }^{24}$ (kindly provided by $\mathrm{G}$ Cossu). Antisera to mouse or rabbit immunoglobulins were from ICN-Cappel. Immunofluorescence assays were performed by fixing the cells directly in the culture vessels and staining them in situ.

BrdUrd incorporation in differentiated muscle cells was assessed by double-immunofluorescence staining for BrdUrd and MHC. Only MHC-positive cells possessing $\geqslant 2$ nuclei were scored. In the vast majority of cases, even in experiments with 6-h BrdUrd pulses, all of the nuclei in a single myotube were concordantly labeled or unlabeled. Rare myotubes displaying discordant nuclei were scored as positive if at least two contiguous nuclei were BrdUrd-positive (usually lightly labeled, having been fixed at the beginning of S-phase). Myogenin expression in MHC-positive cells was determined as above, with the antibody to myogenin replacing that to BrdUrd. All nuclei within a single myotube were always concordant with regard to myogenin expression. In all immunofluorescence procedures, nuclei were counterstained with Hoechst 33258.

\section{TUNEL staining and Western blotting}

Diluted supernatant from an MF20 hybridoma culture was used to detect MHC by Western analysis. Blots were developed using the ECL 
kit (Amersham). TUNEL assays were performed on cytocentrifuge slides. Infected myotubes were detached with trypsin at the appropriate time and counted. Approximately 20000 myotubes were cytocentrifuged onto a microscope slide, allowed to dry, fixed in $4 \%$ buffered formaldehyde, permeabilized in $0.25 \%$ Triton X-100, and TUNEL stained using a commercial kit (Oncor).

\section{Cytofluorimetry}

For cell cycle analysis, Ara-C-purified myocytes were incubated overnight at $4^{\circ} \mathrm{C}$ in phosphate-buffered saline containing $100 \mu \mathrm{g} / \mathrm{ml}$ propidium iodide, $200 \mu \mathrm{g} / \mathrm{ml}$ RNAse, and $0.2 \%$ Triton X-100 and analyzed with an EPICS XL cytofluorimeter (Coulter).

\section{Time-lapse videomicroscopy}

Ara-C-purified quail myotubes were prepared in a $25-\mathrm{cm}^{2}$, tissue culture flask. The myotubes were infected with dl520 and maintained in SF medium for $24 \mathrm{~h}$. The myotubes were then switched to medium containing $7 \%$ fetal bovine serum and $25 \mathrm{mM}$ HEPES to stabilize $\mathrm{pH}$ in the absence of gas exchanges. The flask was then sealed and subjected to time-lapse, phase-contrast videomicroscopy on the heated stage of an inverted microscope.

\section{Acknowledgments}

The authors are indebted to their colleagues who donated indispensable materials to carry out the present work. The authors thank F Tato' and S Soddu for critically reviewing the manuscript. This study was supported by Telethon grant no. 761 and by an AIRC grant to M Crescenzi, who gratefully acknowledges these contributions.

\section{References}

1. Okazaki K and Holtzer $H$ (1966) Myogenesis: fusion, myosin synthesis, and the mitotic cycle. Proc. Natl. Acad. Sci. USA 56: 1484-1490

2. Fogel $M$ and Defendi $V$ (1967) Infection of muscle cultures from various species with oncogenic DNA viruses (SV40 and polyoma). Proc. Natl. Acad. Sci. USA 58: 967-973

3. Yaffe D and Gershon D (1967) Multinucleated muscle fibres: induction of DNA synthesis and mitosis by polyoma virus infection. Nature 215: 421-424

4. Graessmann A, Graessmann M and Fogel M (1973) The relationship of polyoma virus-induced tumor $(T)$ antigen to activation of DNA synthesis in rat myotubes. Dev. Biol. 35: 180-186

5. Endo T and Nadal-Ginard B (1998) SV40 large T antigen induces reentry of terminally differentiated myotubes into the cell cycle. In Cellular and molecular biology of muscle development, Kedes LH and Stockdale FE, (eds). (New York: Alan R. Liss, Inc.) pp. 95-104

6. Crescenzi M, Soddu S and Tato' $F$ (1995) Mitotic cycle reactivation in terminally differentiated cells by adenovirus infection. J. Cell. Physiol. 162: 26-35
7. Le Gal La Salle G, Robert JJ, Berrard S, Ridoux V, Stratford-Perricaudet LD, Perricaudet $M$ and Mallet $J$ (1993) An adenovirus vector for gene transfer into neurons and glia in the brain. Science 259: 988-990

8. Quantin B, PerricaudetLD, Tajbakhsh Sand Mandel J-L(1992) Adenovirus as an expression vector in muscle cells in vitro. Proc. Natl. Acad. Sci. USA 89: 2581 2584

9. Crescenzi M, Soddu S, Sacchi A and Tato'F(1995) Adenovirus infection induces reentry into the cell cycle of terminally differentiated skeletal muscle cells. Ann. N.Y. Acad. Sci. 752: $9-18$

10. Kirshenbaum LA and Schneider MD (1995) Adenovirus E1A represses cardiac gene transcription and reactivates DNA synthesis in ventricular myocytes, via alternative pocket protein- and p300-binding domains. J. Biol. Chem. 270: $7791-7794$

11. Tiainen M, Spitkovsky D, Jansen-Dürr P, Sacchi A and Crescenzi M (1996) Expression of $\mathrm{E} 1 \mathrm{~A}$ in terminally differentiated muscle cells reactivates the cell cycle and suppresses tissue-specific genes by separable mechanisms. Mol. Cell. Biol. 16: 5302-5312

12. Tiainen M, Pajalunga D, Ferrantelli F, Soddu S, Salvatori G, Sacchi A and Crescenzi M (1996) Terminally differentiated skeletal myotubes are not confined in $G_{0}$, but can enter $G_{1}$ upon growth factor stimulation. Cell Growth Diff. 7: 1039 1050

13. Webster KA, Muscat GE and Kedes L (1988) Adenovirus E1A products suppress myogenic differentiation and inhibit transcription from muscle-specific promoters. Nature 332: 553-557

14. Caruso M, Martelli F, Giordano A and Felsani A (1993) Regulation of MyoD gene transcription and protein function by the transforming domains of the adenovirus E1A oncoprotein. Oncogene 8: 267-278

15. Sandmöller A, Meents $H$ and Arnold $H H$ (1996) A novel E1A domain mediates skeletal-muscle-specific enhancer repression independently of pRb and $\mathrm{p} 300$ binding. Mol. Cell. Biol. 16: 5846-5856

16. Alema' $S$ and Tato' $F$ (1994) Oncogenes and muscle differentiation: multiple mechanisms of interference. Sem. Cancer Biol. 5: 147-156

17. Rieder CL, Khodjakov A, Paliulis LV, Fortier TM, Cole RW and Sluder G (1997) Mitosis in vertebrate somatic cells with two spindles: implications for the metaphase/anaphase transition checkpoint and cleavage. Proc. Natl. Acad. Sci. USA 94: 5107-5112

18. Endo T and Nadal-Ginard B (1998) Reversal of myogenic terminal differentiation by SV40 large T antigen results in mitosis and apoptosis. J. Cell. Sci. 111: 10811093

19. Teodoro JG and Branton PE (1997) Regulation of apoptosis by viral gene products. J. Virol. 71: 1739-1746

20. Yaffe D and Saxel $O$ (1977) Serial passaging and differentiation of myogenic cells isolated from dystrophic mouse muscle. Nature 270: 725-727

21. Blau HM, Pavlath GK, Hardeman EC, Chiu C-P, Silberstein L, Webster SG, Miller SC and Webster C (1985) Plasticity of the differentiated state. Science 230: $758-766$

22. Tato F, Alema S, Dlugosz A, Boettiger D, Holtzer H, Cossu G and Pacifici M (1983) Development of 'revertant' myotubes in cultures of Rous sarcoma virus transformed avian myogenic cells. Differentiation 24: 131-139

23. Bader D, Masaki Tand Fischman DA (1982) Immunochemical analysis of myosin heavy chain during avian myogenesis in vivo and in vitro. J. Cell Biol. 95: 763770

24. Wright WE, Dac-Korytko I and Farmer K (1996) Monoclonal antimyogenin antibodies define epitopes outside the $\mathrm{bHLH}$ domain where binding interferes with protein-protein and protein-DNA interactions. Dev. Genet 19: 131-138 